\title{
Síndrome neuroléptica maligna de paciente em uso de olanzapina
}

\author{
Neuroleptic malignant syndrome in patient using olanzapine
}

Fabrício Lins de Medeiros', Arthur Guerra de Andrade',2, Vivian Yuri Hiroce', Tânia Corrêa de Toledo Ferraz Alves 1,2

\begin{abstract}
RESUMO
A síndrome neuroléptica maligna (SNM) é uma reação idiossincrásica rara, extremamente grave e potencialmente fatal ao uso de antipsicóticos, tanto típicos quanto atípicos, bem como drogas de ação dopaminérgica. O diagnóstico fundamenta-se em critérios clínicos e laboratoriais e exclusão de outras condições médicas gerais ou psiquiátricas que melhor expliquem os sintomas. Segundo o DSM-IV, os principais critérios são rigidez muscular grave e temperatura elevada, associadas ao uso de medicação antipsicótica. Foi relatado um caso de paciente com 30 anos manifestando história de transtorno afetivo bipolar, que apresentou sinais e sintomas consistentes com SNM, após três semanas de tratamento com olanzapina. Esse relato visa a discutir o risco da SNM ao uso de antipsicóticos atípicos, bem como a importância de diagnóstico precoce e intervenção imediata.
\end{abstract}

\begin{abstract}
Neuroleptic malignant syndrome (NMS) is an idiosyncratic, serious and potentially fatal disorder observed in patients who receive treatment with neuroleptics, typical and atypical, as well as medications with dopaminergic effects. The diagnosis is based on clinical and laboratory criteria and the exclusion of other general medical or psychiatric conditions that could best explain the symptoms. The main criteria according to DSM-IV are severe rigidity and fever associated with the use of antipsychotic medication. We present a case of a 30-year-old female with history of bipolar affective disorder that developed signs and symptoms consistent with NMS after three weeks of treatment with Olanzapine. This case aims to address the risk of NMS associated atypical antipsychotic, as well as the importance of an early diagnosis and immediate intervention.
\end{abstract}

\section{Keywords}

Atypical antipsychotics, neuroleptic malignant syndrome, olanzapine.

\section{INTRODUÇÃO}

A olanzapina é um antipsicótico atípico da classe das tienobenzodiazepinas, que exerce antagonismo pós-sináptico em quase todos os receptores centrais dopaminérgicos (D) $D_{1-4^{\prime}}$ serotoninérgicos $(5 \mathrm{Ht}) 2 \mathrm{~A} / 2 \mathrm{C}$, adrenérgicos, muscarínicos $M_{1-5}$ e histaminérgicos $H_{1}$. Possui fracas propriedades de bloqueio $D_{2}$ e o seu bloqueio serotoninérgico $5 \mathrm{Ht} 2 \mathrm{~A}$ é cerca de oito vezes mais intenso que o bloqueio dopaminérgico $D_{2}{ }^{\text {. }}$.

A síndrome neuroléptica maligna (SNM) é uma reação idiossincrásica rara, muitíssimo grave e potencialmente fatal ao uso de antipsicóticos, tanto típicos quanto atípicos, bem como drogas de ação dopaminérgica² ${ }^{2}$ A incidência da SNM

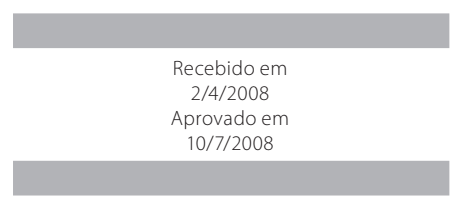

1. Departamento de Psiquiatria e Psicologia Médica da Faculdade de Medicina do ABC (FMABC). 2. Departamento de Psiquiatria da Faculdade de Medicina da Universidade de São Paulo (FMUSP).

Endereço para correspondência: Fabrício Lins de Medeiros Av. Lauro Gomes, 2000, Vila Sacadura Cabral - 09060-870 - Santo André, SP E-mail:emfabricio@yahoo.com.br 
relacionada com antipsicóticos típicos varia entre 0,02\% a $2,46 \%$, mas não é bem estabelecida para antipsicóticos atípicos $^{3}$. Não obstante o diagnóstico precoce, a SNM é fatal entre $10 \%$ a $20 \%$ dos casos ${ }^{1}$. Os fatores de risco relacionados à SNM são episódio prévio de SNM, uso de antipsicóticos de alta potência, titulação rápida da dose, administração parenteral, desidratação, uso concomitante de lítio, doença afetiva ou neurológica prévia, exaustão física e agitação psicomotora 4 .

Não existe definição universalmente aceita para a SNM. O diagnóstico fundamenta-se em critérios clínicos e laboratoriais, bem como da exclusão de outras condições médicas gerais ou psiquiátricas que mais bem explicariam os sintomas ${ }^{5}$. Os critérios do Diagnostic and Statistical Manual of Mental Disorders IV (DSM-IV) ${ }^{6}$ incluem rigidez muscular grave e elevação da temperatura e, pelo menos, outros dois sintomas entre diaforese, disfagia, tremor, incontinência, alterações no nível de consciência (confusão ao coma), mutismo, taquicardia, pressão arterial elevada ou instável, leucocitose e evidências laboratoriais de lesão muscular (por exemplo, creatinofosfoquinase [CPK] elevada), além da exclusão de outras condições médicas ou intoxicação por alguma substância ${ }^{6}$.

O presente relato visa a discutir o risco de ocorrência da SNM com uso de antipsicótico atípico, particularmente a olanzapina, assim como a importância do diagnóstico precoce e a intervenção imediata.

\section{CASO CLÍNICO}

Paciente A. R., 30 anos, em acompanhamento psiquiátrico por transtorno afetivo bipolar tipo I há 13 anos e com cinco internações psiquiátricas prévias durante episódios maníacos com sintomas psicóticos. Após receber alta de sua última internação psiquiátrica, que teve duração de 40 dias, foi admitida no serviço de emergência após cinco dias com queixa de apatia e lentificação motora. Não tinha antecedente de SNM, negava uso de álcool ou qualquer outra droga. Havia iniciado uso de olanzapina $5 \mathrm{mg} /$ dia durante sua internação anterior, cerca de 30 dias antes do início do quadro, com aumento progressivo até $25 \mathrm{mg} / \mathrm{dia}$. Ao exame de entrada apresentava hipertermia $\left(37,8^{\circ} \mathrm{C}\right)$, hipotensão arterial (pressão arterial $90 \times 60 \mathrm{mmHg}$ ), pulso 84 bpm, desidratada e rebaixamento de nível de consciência (Glasgow 13). Em 24 horas apresentou piora do estado geral, elevação da temperatura $\left(40,1^{\circ} \mathrm{C}\right)$, rigidez de membros superiores e inferiores e negativismo ativo. Os exames de entrada indicavam leucocitose com 18.100 leucócitos $/ \mu \mathrm{L} / \mathrm{mm}^{3}$ (valores normais de 4.300 a $10.800 / \mathrm{mm}^{3}$ ), hemoglobina e plaquetas normais em número e morfologia; creatinina 1,6 mg/dL (valores normais de 0,6 a 1,2 mg/dL); sódio $141 \mathrm{mEq} / \mathrm{l}$ (135 a
$145 \mathrm{mEq} / \mathrm{l}) ;$ potássio 4,1 mEq/l (3,5 a 5,0 mEq/l); alanina (ALT) $27 \mathrm{U} / \mathrm{I}(1$ a $21 \mathrm{U} / \mathrm{I})$; aspartato (AST) $32 \mathrm{U} / \mathrm{I}$ (7 a $27 \mathrm{U} / \mathrm{I}) ; \mathrm{TSH}$ $1,3 \mu \mathrm{UI} / \mathrm{ml}(0,5$ a $5,0 \mu \mathrm{UI} / \mathrm{ml}) ; \mathrm{T} 4 \mathrm{~L}$ 1,1 ng/dL (0,6 a 1,54 ng/dl); e aumento dos níveis séricos de CPK para 2.967 U/I (96 a 140 U/I). Tomografia computadorizada de crânio sem evidência de lesões expansivas ou hemorragia, e líquido cefaIorraquidiano sem anormalidades. Feita a hipótese diagnóstica de síndrome extrapiramidal medicamentosa - catatonia - SNM causada pelo uso de olanzapina. Foram descartadas outras causas orgânicas, como presença de infecção urinária, insuficiência renal aguda ou infarto agudo do miocárdio. A paciente tinha antecedente de uso de outros antipsicóticos, como haloperidol $10 \mathrm{mg} / \mathrm{dia}$, biperideno $2 \mathrm{mg} / \mathrm{dia}$ e clorpromazina 200 mg/dia no passado. Estava em uso atual de olanzapina 25 mg/dia (iniciada há 30 dias na última internação), carbonato de lítio $1.500 \mathrm{mg} / \mathrm{dia}$, carbamazepina 600 mg/dia e clonazepam 4 mg/dia. A abordagem terapêutica incluiu suporte clínico na unidade de terapia intensiva (UTI) e suspensão do agente (no caso a olanzapina). Na evolução, a paciente apresentou remissão do quadro da SNM, com normalização dos parâmetros laboratoriais em 13 dias (leucócitos: $9.100 / \mathrm{mm}^{3}$; ALT: $48 \mathrm{U} /$; AST: $60 \mathrm{U} / \mathrm{l}$ - exames na alta hospitalar). Após a resolução do quadro, optou-se pela introdução de quetiapina 700 mg/dia, ácido valpróico 750 mg/dia e clonazepam 2 mg/dia, para manutenção da estabilização do transtorno afetivo bipolar.

\section{DISCUSSÃO}

A SNM é principalmente causada pelo bloqueio de receptores dopaminérgicos $D_{2}$ no trato nigroestriatal, na via mesocortical e no núcleo hipotalâmico. No entanto, a ocorrência da SNM com antipsicóticos atípicos e de baixa potência sugere outros fatores envolvidos na sua patofisiologia7. A maioria dos casos relatados, entre $80 \%$ a $90 \%$, ocorreu 30 dias antes do início ou por ocasião do aumento da dose do agente, embora em alguns casos tenham ocorrido até após um ano de uso regular da medicação'. O caso relatado manifestou os primeiros sintomas após 40 dias da introdução do agente, e após cerca de 20 dias do aumento da dose para $25 \mathrm{mg} / \mathrm{dia}$.

Entre os fatores de risco para a SNM, a paciente apresentava uso concomitante de carbonato de lítio e estava desidratada na admissão, além de ter diagnóstico de transtorno afetivo. No primeiro dia de internação apresentou os principais sintomas relacionados com a SNM: rigidez muscular grave e aumento da temperatura corporal $\left(40,1^{\circ} \mathrm{C}\right)$ e outros quatro critérios, segundo o DSM IV (alteração do nível de consciência, mutismo, leucocitose importante e evidências laboratoriais de lesão muscular). 
Para o diagnóstico diferencial da SNM, diversas situações clínicas devem ser consideradas, sendo diagnóstico de exclusão em pacientes com alteração do estado mental, febre e hipertonia. Entre os principais diagnósticos diferenciais estão infecções, principalmente pneumonias, acidente vascular cerebral, distúrbios hidroeletrolíticos, endocrinopatias como tempestade tireoidiana e feocromocitoma, insuficiência renal aguda, síndrome serotoninérgica e hipertermia maligna ${ }^{8}$. Os referidos diagnósticos diferenciais foram excluídos por meio da história, do exame físico e dos exames complementares (radiografia de tórax, tomografia computadorizada de crânio e líquido cefalorraquidiano dentro dos padrões de normalidade).

O tratamento da SNM consiste da retirada do agente e de medidas de suporte clínico, de preferência em UTI. Para tratamento específico da SNM podem ser utilizados os agonistas dopaminérgicos (como a bromocriptina e a amantadina), que por sua ação direta reverteriam o bloqueio dos receptores dopaminérgicos, ou o dantrolene, que atua como relaxante muscular. Além da abordagem medicamentosa, pode-se utilizar a eletroconvulsoterapia. A dose inicial recomendada de bromocriptina é de 2,5 a $5 \mathrm{mg}$, via oral, três vezes ao dia, que pode ser aumentada até o total de 30 a $45 \mathrm{mg}^{5,9}$. Já o dantrolene pode ser utilizado via oral ou endovenosa, na dose de 1 a $10 \mathrm{mg} / \mathrm{kg} / \mathrm{dia}^{10}$. No caso relatado, o tratamento foi realizado apenas com suporte clínico, havendo completa remissão dos sintomas após duas semanas, tempo médio segundo a literatura².

A retirada dos neurolépticos deve ser imediata após suspeita de SNM, como forma de evitar complicações, e constitui problema no caso de formulações de depósito. As medidas de suporte clínico utilizadas incluem hidratação, suporte ventilatório, cardiovascular, renal e nutricional adequados, preferencialmente em UTI, como no caso relatado, e de ventilação mecânica para proteção de vias respiratórias. As principais complicações da SNM a serem evitadas são distúrbios respiratórios e insuficiência renal, associadas com coagulação intravascular disseminada e rabdomiólise, e instabilidade hemodinâmica8 .

Deve-se evitar a reintrodução do antipsicótico associado ao surgimento do episódio de SNM, e sempre optar por outro antipsicótico cujo potencial de induzir a SNM seja reconhecidamente baixo, levando-se em consideração a relação risco/benefício da medicação. O acompanhamento com exames laboratoriais para monitorizar eventuais alterações é essencial depois da troca da medicação². Após mais duas semanas, foi reiniciado o tratamento para estabilização do humor com uso de quetiapina (700 mg/dia), com boa resposta e sem recorrência da SNM.

\section{CONCLUSÃO}

Concluiu-se que o risco de SNM deve ser considerado mesmo em uso de antipsicóticos atípicos, e que a suspeita diagnóstica e o suporte clínico são importantes para o prognóstico do paciente. Outro ponto importante a se considerar é o uso de antipsicóticos atípicos em associação com lítio, tendo-se em vista o maior risco de SNM nesta associação e as graves repercussões da SNM, embora seja uma entidade rara.

\section{REFERÊNCIAS}

1. Kogoj A, Velikonja I. Olanzapine induced neuroleptic malignant syndrome-a case review. Hum Psychopharmacol Clin Exp. 2003;18:301-9.

2. Fekadua A, Bisson J. Neuroleptic malignant syndrome: diagnostic and therapeutic dilemmas. Behav Neurol. 2005;16:9-13.

3. Nielsen J, Bruhn AM. Atypical neuroleptic malignant syndrome caused by olanzapine. Acta Psychiatr Scand. 2005;112:238-40

4. Berardi D, Dell'Atti M, Amore M, De Ronchi D, Ferrari G. Clinical risk factors for neuroleptic malignant syndrome. Hum Psychopharmacol. 2002;17:99-102.

5. Seitz DP. Diagnostic uncertainty in a case of neuroleptic malignant syndrome. CJEM. 2005;7(4):266-72.

6. American Psychiatric Association (APA). Manual diagnóstico e estatístico de transtornos mentais (DSM-IV-TR). $4^{\mathrm{a}}$ ed. 1994.

7. Kontaxakis VP, Havaki-kontaxaki BJ, Christodoulou NG, Paplos KG, Christodoulou GN. Olanzapine-associated neuroleptic malignant syndrome: is there an overlap with the serotonin syndrome? Ann Gen Hosp Psychiatry. 2003;2:10.

8. Nicholson D, Chiu W. Neuroleptic malignant syndrome. Geriatrics. $2004 ; 59(8): 36-40$.

9. Susman VL. Clinical management of neuroleptic malignant syndrome. Psychiatr $\mathbf{Q}$. 2001;72(4):325-36.

10. Krause T, Gerbershagen MU, Fiege M, Weisshorn R, Wappler F. Dantrolene-a review of its pharmacology, therapeutic use and new developments. Anaesthesia. 2004;59(4):364-73. 